\title{
The heat equation with inhomogeneous Dirichlet boundary conditions
}

\author{
M. VAN DEN Berg AND P. B. GILKeY ${ }^{1}$
}

\begin{abstract}
We establish the existence of an asymptotic expansion for the heat content asymptotics with inhomogeneous Dirichlet boundary conditions and compute the first 5 coefficients in the asymptotic expansion.
\end{abstract}

\section{Introduction.}

Let $M$ be a smooth compact Riemannian manifold of dimension $m$ with smooth boundary $\partial M$. For $\phi \in C^{\infty}(\partial M)$, let $\mathcal{E}(\phi)(t)$ be the total heat energy content of $M$ where the initial temperature is 0 and where the boundary of $M$ is kept at temperature $\phi$; see $\S 1$ for a more precise definition. Let $\Phi_{\phi}$ be the harmonic function with boundary value $\phi$. It is well known that for large time the temperature profile of $M$ approaches $\Phi_{\phi}$, and that

$$
\lim _{t \rightarrow \infty} \mathcal{E}(\phi)(t)=\int_{M} \Phi_{\phi}
$$

This is a globally defined invariant which is not locally computable. For short time the total heat content $\mathcal{E}(\phi)(t)$ has an asymptotic expansion. It is somewhat surprising in contrast to the large time behaviour that the coefficients in that expansion are locally computable.

Theorem 0.1. As $t \downarrow 0, \mathcal{E}(\phi)(t) \cong \sum_{n \geq 1} \mathcal{B}_{n}(\phi) t^{n / 2}$. There exist locally defined geometric invariants $B_{n}$ on the boundary so that $\mathcal{B}_{n}(\phi)=\int_{\partial M} \phi \cdot B_{n}$.

The coefficients $\mathcal{B}_{n}(\phi)$ express the net heat flow into and out of the manifold over the boundary $\partial M$. The case $\phi=1$ has particular geometrical significance since the coefficients are then invariants of the boundary of $M$.

\footnotetext{
${ }^{1}$ EPSRC grant K85391 (UK)
} 
The classical analytical tools to study these inhomogeneous problems rely on explicit formulas for the Dirichlet heat kernel, and are available in a few special cases only. See, for example, Carslaw and Jaeger [4, §9.11-9.12] for the ball in $\mathbb{R}^{3}$. In Theorem 0.2 below, we give the first five asymptotic coefficients; we will use methods of invariance theory to derive these formulas. Let $L$ be the second fundamental form on the boundary, let $R$ be the Riemann curvature tensor of $M$ and let $\rho_{i j}:=R_{i k k j}$ be the Ricci tensor. Let ';' and ':' denote covariant differentiation with respect to the Levi-Civita connections of $M$ and of $\partial M$ respectively. We choose a local orthonormal frame $\left\{e_{1}, \ldots, e_{m}\right\}$ for the tangent bundle of $M$ restricted to the boundary so that $e_{m}$ is the inward unit normal. Let indices $a, b, c$ etc. range from 1 through $m-1$. We adopt the Einstein convention and sum over repeated indices.

Theorem 0.2. The geometric invariants $B_{1}, \ldots, B_{4}$ in Theorem 0.1 are given by

(1) $\quad B_{1}=\frac{2}{\sqrt{\pi}}$.

(2) $\quad B_{2}=-\frac{1}{2} L_{a a}$.

(3) $\quad B_{3}=\frac{1}{6 \sqrt{\pi}}\left\{L_{a a} L_{b b}-2 L_{a b} L_{a b}-2 \rho_{m m}\right\}$.

(4) $\quad B_{4}=\frac{1}{32}\left\{2 L_{a b} L_{a b} L_{c c}-4 L_{a b} L_{a c} L_{b c}+2 R_{a m b m} L_{a b}-2 R_{a b c b} L_{a c}\right.$

$$
\left.-\rho_{i i ; m}-2 L_{a b: a b}\right\} \text {. }
$$

Moreover

(5)

$$
\begin{aligned}
\mathcal{B}_{5}(1)= & -\frac{1}{240 \sqrt{\pi}} \int_{\partial M}\left\{8 \rho_{m m ; m m}-8 L_{a a} \rho_{m m ; m}+16 L_{a b} R_{a m m b ; m}\right. \\
& -4 \rho_{m m}^{2}+16 R_{a m m b} R_{a m m b}-4 L_{a a} L_{b b} \rho_{m m}-8 L_{a b} L_{a b} \rho_{m m} \\
& +64 L_{a b} L_{a c} R_{m b c m}-16 L_{a a} L_{b c} R_{m b c m}-8 L_{a b} L_{a c} R_{b d d c} \\
& -8 L_{a b} L_{c d} R_{a c b d}+4 R_{a b c m} R_{a b c m}+8 R_{a b b m} R_{a c c m}-16 L_{a a: b} R_{b c c m} \\
& -8 L_{a b: c} L_{a b: c}+L_{a a} L_{b b} L_{c c} L_{d d}-4 L_{a a} L_{b b} L_{c d} L_{c d}+4 L_{a b} L_{a b} L_{c d} L_{c d} \\
& \left.-24 L_{a a} L_{b c} L_{c d} L_{d b}+48 L_{a b} L_{b c} L_{c d} L_{d a}\right\} .
\end{aligned}
$$

Here is a brief guide to the paper. In $\S 1$, we prove Theorem 0.1 . We first establish the existence of the asymptotic expansion and then prove the 
coefficients are locally computable. In $\S 2$, we derive Theorem 0.2 (1)-(4) from previously known results for the homogeneous case. The remainder of the paper is devoted to the proof of Theorem $0.2(5)$. In $\S 3$, we establish some product formulas. In $\S 4$, we use these formulas to compute $\mathcal{B}_{5}$ and complete the proof of Theorem 0.2 .

\section{Heat Content Asymptotics.}

We begin by giving rigorous definitions of the heat content functions with which we shall be working. Let $D$ be a scalar operator of Laplace type. For $\Phi \in C^{\infty}(M)$ and $\phi \in C^{\infty}(\partial M)$, let $u_{\Phi}(x ; t)$ and $u_{\phi}(x ; t)$ mapping $M \times[0, \infty)$ to $\mathbb{R}$ be the unique solutions of the equations:

$$
\begin{aligned}
& \partial_{t} u_{\Phi}=-D u_{\Phi}, u_{\Phi}(x ; 0)=\Phi(x) \text { for } x \in M \\
& u_{\Phi}(y ; t)=0 \text { for } y \in \partial M, t>0
\end{aligned}
$$

and

$$
\begin{aligned}
& \partial_{t} u_{\phi}=-D u_{\phi}, u_{\phi}(x ; 0)=0 \text { for } x \in M \\
& u_{\phi}(y ; t)=\phi(y) \text { for } y \in \partial M, t>0 .
\end{aligned}
$$

The function $u_{\Phi}$ solves the heat equation with initial temperature $\Phi$ and zero (Dirichlet) boundary condition and the function $u_{\phi}$ solves the heat equation with initial temperature 0 and inhomogeneous boundary condition defined by $\phi$. Let $\tilde{\Phi}$ be an auxiliary test function. Let

$$
E(\Phi, \tilde{\Phi}, D)(t):=\int_{M} u_{\Phi} \tilde{\Phi} \text { and } \mathcal{E}(\phi, \tilde{\Phi}, D)(t):=\int_{M} u_{\phi} \tilde{\Phi} .
$$

Let $\Delta$ be the scalar Laplacian. We recover the function $\mathcal{E}(\phi)$ by setting $D=\Delta$ and $\tilde{\Phi}=1$ in equation (1.2).

Lemma 1.1. (1) As $t \downarrow 0, E(\Phi, \tilde{\Phi}, D)(t) \cong \sum_{n \geq 0} \beta_{n}(\Phi, \tilde{\Phi}, D) t^{n / 2}$. There exist local invariants $\beta_{n}^{\partial M}(\Phi, \tilde{\Phi}, D) \in C^{\infty}(\partial M)$ so that

$$
\beta_{2 k-1}(\Phi, \tilde{\Phi}, D)=\int_{\partial M} \beta_{2 k-1}^{\partial M}(\Phi, \tilde{\Phi}, D)
$$

and so

$$
\beta_{2 k}(\Phi, \tilde{\Phi}, D)=\int_{\partial M} \beta_{2 k}^{\partial M}(\Phi, \tilde{\Phi}, D)+(-1)^{k} \int_{M} D^{k} \Phi(x) \tilde{\Phi}(x) d x / k ! .
$$


(2) $\beta_{0}(\Phi, \tilde{\Phi}, D)=\int_{M} \Phi(x) \tilde{\Phi}(x) d x$ and $\beta_{1}(\Phi, \tilde{\Phi}, D)=-\frac{2}{\sqrt{\pi}} \int_{\partial M} \Phi \tilde{\Phi}$.

(3) If $\left.\Phi\right|_{\partial M}=0$, then $\beta_{n}(\Phi, \tilde{\Phi}, D)=-\frac{2}{n} \beta_{n-2}(D \Phi, \tilde{\Phi}, D)$.

(4) If $\tilde{D}$ is the formal adjoint of $D$, then $\beta_{n}(\Phi, \tilde{\Phi}, D)=\beta_{n}(\tilde{\Phi}, \Phi, \tilde{D})$.

(5) $\left.\partial_{\epsilon}\right|_{\epsilon=0} \beta_{n}(\Phi, \tilde{\Phi}, D-\epsilon)=\beta_{n-2}(\Phi, \tilde{\Phi}, D)$.

(6) Let $M$ be the unit disk in $\mathbb{R}^{m}$ for $m \geq 2$. Then

$$
\beta_{5}(1,1, \Delta)=\frac{1}{240 \sqrt{\pi}}(m-1)(m-3)(m+3)(m-7) \operatorname{vol}\left(S^{m-1}\right) .
$$

Proof. We refer to [2, Lemma 1.3] and to [5, equation 2.17] for the proof of (1), we refer to [2, Theorem 1.1] for the proof of (2), we refer to [2, Lemma $3.2]$ for the proof of $(3,4)$, we refer to [5, Lemma 2.2] for the proof of (5), and we refer to [2, Theorem 4.2] for the proof of (6).

We can now establish the existence of the asymptotic series given in Theorem 0.1 for inhomogeneous boundary conditions. Let $\Phi=\Phi_{\phi}$ be a harmonic function on $M$ so that $\left.\Phi\right|_{\partial M}=\phi$. Then $\Phi-u_{\Phi}$ satisfies the equations defining $u_{\phi}$ so $\Phi-u_{\Phi}=u_{\phi}$.

Consequently $\mathcal{E}(\phi, 1, \Delta)(t)=\int_{M} \Phi-E(\Phi, 1, \Delta)(t)$. Thus the asymptotic series for $E(\Phi, 1, \Delta)(t)$ given in Lemma 1.1 shows there is a corresponding asymptotic series for $\mathcal{E}$ where

$$
\mathcal{B}_{n}(\phi)=-\beta_{n}(\Phi) \text { for } n \geq 1 .
$$

The constant term in the asymptotic expansion $\beta_{0}$ is cancelled by $-\int_{M} \Phi$ so the asymptotic expansion for $\mathcal{E}$ begins with $n=1$ and not $n=0$.

The formalism which expresses $\mathcal{B}_{n}(\phi)=-\beta_{n}(\Phi)$ a priori permits the normal derivatives of $\Phi$ to enter into the formulas; this would involve the Dirichlet to Neumann operator which is not local. Thus to complete the proof of Theorem 0.1, we must show that the normal derivatives of $\Phi$ do not appear.

We now recall some local geometry of operators of Laplace type. Let indices $\nu, \mu$ range from 1 through $m$ and index a local coordinate frame on the interior of $M$. Let $\Gamma$ be the Christoffel symbols of the Levi-Civita 
connection of $M$. Let $D=-\left(g^{\nu \mu} \partial_{\nu} \partial_{\mu}+P^{\nu} \partial_{\nu}+Q\right)$ be an operator of Laplace type on $C^{\infty}(M)$. Let $\Gamma$ be the Christoffel symbols. We refer to [6, Theorem $2.1]$ for the proof of the following Lemma.

Lemma 1.2. There exists a unique connection $\nabla$ and smooth potential $\mathcal{P}$ so that $D \Phi=-g^{\nu \mu} \Phi_{; \nu \mu}-\mathcal{P}$. We have

$$
\mathcal{P}=Q-g^{\nu \mu}\left(\partial_{\mu} \omega_{\nu}+\omega_{\nu} \omega_{\mu}-\omega_{\sigma} \Gamma_{\nu \mu}^{\sigma}\right),
$$

where $\omega_{\nu}=\frac{1}{2} g_{\nu \mu}\left(P^{\mu}+g^{\sigma \gamma} \Gamma_{\sigma \gamma}{ }^{\mu}\right)$ is the connection 1 form of $\nabla$.

We begin the proof that the invariants $\mathcal{B}_{n}$ are locally computable by constructing a 4 -fold decomposition of the invariants $\beta_{n}^{\partial M}$ of Lemma 1.1 using the parity of the number of normal derivatives of $\Phi$ and $\tilde{\Phi}$. We have to be careful with the decomposition since we could use the identity $\Phi_{; a a}=$ $\Phi_{: a a}-L_{a a} \Phi_{; m}$ to mix even and odd parities. We consider bilinear partial differential operators $A(\Phi, \tilde{\Phi})$ which are invariantly defined and which only involve tangential covariant derivatives of $\Phi$ and $\tilde{\Phi}$ using the restriction of the connections $\nabla$ and $\tilde{\nabla}$ to the boundary of $M$ and using the Levi-Civita connection of the boundary. Thus, for example, we would permit expressions of the form $\Phi_{: a} \tilde{\Phi}_{: a}$ or $L_{a a} \Phi \tilde{\Phi}$ but would not permit expressions $\Phi_{; a} \tilde{\Phi}_{; a}$ or $L_{a a} \Phi_{; m} \tilde{\Phi}$. Let $\mathcal{A}$ be the vector space of all such operators. Let $\Phi^{(0)}:=\Phi$ and $\Phi^{(1)}:=\Phi_{; m}$. For $p=0,1$ and $q=0,1$, let $\mathcal{V}_{p, q}$ be the vector space of all operators of the form

$$
B_{p, q}(\Phi, \tilde{\Phi}, D)=\sum_{a, b} A_{a, b}\left(\left(D^{a} \Phi\right)^{(p)},\left(\tilde{D}^{b} \tilde{\Phi}\right)^{(q)}\right)
$$

for $A_{a, b} \in \mathcal{A}$. Instead of using the variables $\Phi_{; m \cdots m}$, we use the variables $D^{a} \Phi$ and $\left(D^{a} \Phi\right)_{; m}$. This permits us to decompose $\beta_{n}^{\partial M}=\sum_{p, q} B_{n, p, q}$ for $B_{n, p, q} \in \mathcal{V}_{p, q}$.

Lemma 1.3. We have $\int_{\partial M} B_{n, 1,0}(\Phi, \tilde{\Phi}, D)=0$.

Proof. We proceed by induction on $n$; the cases $n=0$ and $n=1$ follow from Lemma 1.1. Choose $F$ and $\tilde{F}$ so that

$$
\begin{aligned}
& \left.D^{a} F\right|_{\partial M}=0,\left.\quad\left(D^{a} F\right)_{; m}\right|_{\partial M}=\left.\left(D^{a} \Phi\right)_{; m}\right|_{\partial M}, \\
& \left.\tilde{D}^{a} \tilde{F}\right|_{\partial M}=\left.\tilde{D}^{a} \tilde{\Phi}\right|_{\partial M} \quad \text { and }\left.\quad\left(\tilde{D}^{a} \tilde{F}\right)_{; m}\right|_{\partial M}=0
\end{aligned}
$$


for all $a$. Then $B_{u, v}(F, \tilde{F}, D)=0$ if $(u, v) \neq(1,0)$ so

$$
\int_{\partial M} \beta_{n-2}^{\partial M}(D F, \tilde{F}, D)=\int_{\partial M} B_{n-2,1,0}(D F, \tilde{F}, D)=0 .
$$

If $n=2 k$, we use Lemma 1.1 to see

$$
\begin{aligned}
\beta_{n}(F, \tilde{F}, D) & =\frac{(-1)^{k}}{k !} \int_{M} D^{k} F \cdot \tilde{F}+\int_{\partial M} B_{n, 1,0}(F, \tilde{F}, D) \\
& =-\frac{1}{k} \beta_{n-2}(F, \tilde{F}, D)=\frac{(-1)^{k}}{k !} \int_{M} D^{k-1}(D F) \cdot \tilde{F}
\end{aligned}
$$

Thus

$$
\int_{\partial M} B_{n, 1,0}(\Phi, \tilde{\Phi}, D)=\int_{\partial M} B_{n, 1,0}(F, \tilde{F}, D)=0 .
$$

The argument is similar if $n$ is odd; the interior integrals are not present.

We can now complete the proof of Theorem 0.1. Let $\phi \in C^{\infty}(\partial M)$. Choose $\Phi$ harmonic with $\left.\Phi\right|_{\partial M}=\phi$. We apply equation (1.3). We have $B_{n, p, 1}(\Phi, 1)=0$ and $A_{a, b}\left(\Delta^{a} \Phi, \Delta^{b} 1\right)=0$ for $(a, b) \neq(0,0)$. By Lemma 1.3, $\int_{\partial M} B_{n, 1,0}(\Phi, 1)=0$. The interior integral if $n \geq 2$ is even vanishes so

$$
\mathcal{B}_{n}(\phi)=-\beta_{n}(\Phi)=-\int_{\partial M} A_{0,0}(\Phi, 1, \Delta)
$$

We integrate by parts tangentially to eliminate any tangential covariant derivatives of $\Phi$ and express

$$
\int_{\partial M}-A_{0,0}(\Phi, 1, \Delta)=\int_{\partial M} B_{n}(g) \Phi=\int_{\partial M} B_{n}(g) \phi . \square
$$

\section{The heat content asymptotics $\mathcal{B}_{n}$ for $n \leq 4$.}

In this section, we prove Theorem 0.2 (1)-(4). We begin by recalling some results for the homogeneous case. Let $\Omega$ be the curvature of the connection defined by $D$. We refer to $[1,2]$ for the proof of the following result; see also $[3,7,8]$.

\section{Lemma 2.1.}

$$
\beta_{0}(\Phi, \tilde{\Phi}, D)=\int_{M} \Phi \tilde{\Phi} .
$$


(2) $\quad \beta_{1}(\Phi, \tilde{\Phi}, D)=-\frac{2}{\sqrt{\pi}} \int_{\partial M} \Phi \tilde{\Phi}$.

$$
\begin{aligned}
\beta_{2}(\Phi, \tilde{\Phi}, D)= & -\int_{M}(D \Phi \cdot \tilde{\Phi})+\int_{\partial M}\left\{\frac{1}{2} L_{a a} \Phi \tilde{\Phi}-\Phi \cdot \tilde{\Phi}_{; m}\right\} \\
\beta_{3}(\Phi, \tilde{\Phi}, D)= & -\frac{2}{\sqrt{\pi}} \int_{\partial M}\left\{\frac { 2 } { 3 } \left(\Phi_{; m m} \tilde{\Phi}+\Phi \tilde{\Phi}_{; m m}\right.\right. \\
& \left.-L_{a a} \Phi_{; m} \tilde{\Phi}-L_{a a} \Phi \tilde{\Phi}_{; m}\right)-\Phi_{: a} \tilde{\Phi}_{: a}+\mathcal{P} \Phi \tilde{\Phi} \\
& \left.+\frac{1}{12}\left(L_{a a} L_{b b}-2 L_{a b} L_{a b}-2 \rho_{m m}\right) \Phi \tilde{\Phi}\right\} \\
\beta_{4}(\Phi, \tilde{\Phi}, D)= & \frac{1}{2} \int_{M}(D \Phi \cdot \tilde{D} \tilde{\Phi})+\frac{1}{32} \int_{\partial M}\left\{16(D \Phi)_{; m} \tilde{\Phi}\right. \\
& +16 \Phi(\tilde{D} \Phi)_{; m}-8 L_{a a} D \Phi \cdot \tilde{\Phi}-8 L_{a a} \Phi \tilde{D} \Phi \\
& +\left(4 \mathcal{P}_{; m}-2 L_{a b} L_{a b} L_{c c}+4 L_{a b} L_{a c} L_{b c}\right. \\
& \left.-2 R_{a m b m} L_{a b}+2 R_{a b c b} L_{a c}+\rho_{i i ; m}+2 L_{a b: a b}\right) \Phi \tilde{\Phi} \\
& \left.-8 L_{a b} \Phi_{: a} \tilde{\Phi}_{: b}-4 \Omega_{a m} \Phi: a \Phi+4 \Omega_{a m} \Phi \cdot \tilde{\Phi}_{: a}\right\}
\end{aligned}
$$

We let $D=\Delta$ be the scalar Laplacian. Then the potential $\mathcal{P}=0$ and the connection $\nabla$ is flat so $\Omega=0$. Let $\Phi$ be a harmonic function with $\left.\Phi\right|_{\partial M}=1$. Set $\tilde{\Phi}=1$. We use equation (1.3) to derive assertions (1), (2), and (4) of Theorem 0.2 from the corresponding assertions of Lemma 2.1. We may use the identity:

$$
0=\Phi_{; i i}=\Phi_{; m m}+\Phi_{: a a}-L_{a a} \Phi_{; m}
$$

to see

$$
\Phi_{; m m}-L_{a a} \Phi_{; m}=\Delta_{\partial M} \Phi=\Delta_{\partial M} \phi=0 .
$$

Assertion (3) of Theorem 0.2 now follows. Note that $\Phi_{; m}$ does not appear in the formula!

\section{Product formulas.}

Let $T^{k}:=S^{1} \times \ldots S^{1}$ be the $k$ dimensional torus with the usual periodic parameters. Let $M_{1}:=\left(r_{0}, r_{1}\right) \times T^{k}$ and $M_{2}:=T^{\ell}$. We use coordinates $(r, u)$ for $u=\left(u_{i}\right)$ on $M_{1}$ and coordinates $v=\left(v_{j}\right)$ on $M_{2}$. We consider a diagonal warped product metric on $M:=M_{1} \times M_{2}$ of the form $d s^{2}=d r^{2}+d u_{i}^{2}+g_{j} d v_{j}^{2}$. We shall assume $g_{j}(r, u, v)$ is identically equal to 1 near $r=r_{1}$ so only the boundary component $\left\{r_{0}\right\} \times T^{k+\ell}$ is relevant. Let $g=\Pi_{j} g_{j}^{1 / 2}$. Then $\Delta_{M}=D_{1}+D_{2}$ for $D_{1}=-g^{-1} \partial_{r} g \partial_{r}-g^{-1} \partial_{i}^{u} g \partial_{i}^{u}$ and $D_{2}=-g^{-1} \partial_{j}^{v} g g_{j}^{-1} \partial_{j}^{v}$. 
Lemma 3.1. If $g=h_{1}(r, u) h_{2}(v)$, then

$$
\beta_{n}\left(1,1, \Delta_{M}\right)=\beta_{n}\left(1, h_{1}, D_{1}\right) \int_{M_{2}} h_{2}(v) d v .
$$

Proof. The operator $D_{1}$ is independent of $v$ by assumption. Let $u_{1}(r, u)$ solve equation (1.1a) for the operator $D_{1}$ on $M_{1}$ with initial condition $\Phi=1$. Then $u_{M}(r, u, v):=u_{1}(r, u)$ solves equation (1.1a) for the operator $\Delta_{M}$ on $M$ with the same initial condition. Since $d v o l_{M}=g d r d u d v$,

$$
\begin{aligned}
E\left(1,1, \Delta_{M}\right)(t) & =\int_{M_{1}} u_{1}(r, u ; t) h_{1}(r, u) d r d u \cdot \int_{M_{2}} h_{2}(v) d v \\
& =E\left(1, h_{1}, D_{1}\right)(t) \int_{M_{2}} h_{2}(v) d v . \square
\end{aligned}
$$

The operator $D_{1}$ of Lemma 3.1 is not a general operator of Laplace type. It is defined on a flat manifold with totally geodesic boundary with flat associated connection; the curvature of $\nabla$ and $\tilde{\nabla}$ vanishes. We compute $\beta_{5}$ for such operators in the following Lemma.

Lemma 3.2. Let $M=\left(r_{0}, r_{1}\right) \times T^{k}$ with the flat product metric.

(1) Let $D$ be an operator of Laplace type on $M$ defining a flat connection. Then

$$
\begin{aligned}
\beta_{5}(\Phi, \tilde{\Phi}, D)= & \frac{1}{15 \sqrt{\pi}} \int_{\partial M}\left\{\mathcal{P}_{; m m} \Phi \tilde{\Phi}-4 \mathcal{P} D \Phi \cdot \tilde{\Phi}-4 \mathcal{P} \Phi \tilde{D} \tilde{\Phi}+\mathcal{P}^{2} \Phi \tilde{\Phi}\right. \\
& -8 D^{2} \Phi \cdot \tilde{\Phi}-8 \Phi \tilde{D}^{2} \tilde{\Phi}-8 D \Phi \cdot \tilde{D} \tilde{\Phi}+5 \mathcal{P}\left(\Phi_{; a a} \tilde{\Phi}+\Phi \tilde{\Phi}_{; a a}\right) \\
& \left.+9 \Phi_{; a a} \tilde{\Phi}_{; b b}+4\left(\Phi_{; m m} \tilde{\Phi}_{; a a}+\Phi_{; a a} \tilde{\Phi}_{; m m}\right)\right\}
\end{aligned}
$$

(2) Let $\tilde{g}=e^{h}$ and $D=-\tilde{g}^{-1}\left(\partial_{r} \tilde{g} \partial_{r}+\sum_{a \leq k} \partial_{x_{k}} \tilde{g} \partial_{x_{k}}\right)$. Then

a) $\mathcal{P}=\mathcal{P}_{r}+\mathcal{P}_{t}$ for

$$
\mathcal{P}_{r}=-\frac{1}{2} \partial_{r}^{2} h-\frac{1}{4}\left(\partial_{r} h\right)^{2}
$$

and

$$
\mathcal{P}_{t}=-\sum_{i}\left(\frac{1}{2} \partial_{i}^{u 2} h+\frac{1}{4}\left(\partial_{i}^{u} h\right)^{2}\right) .
$$

b) $\beta_{5}(1, \tilde{g}, D)=\frac{1}{240 \sqrt{\pi}} \int_{\partial M}\left\{\left(16 \mathcal{P}_{r ; m m}+16 \mathcal{P}_{t ; m m}+16 \mathcal{P}_{r}^{2}\right) \tilde{g}\right\}$. 
Proof. One can use dimensional analysis to see that the local formulas $\beta_{n}^{\partial M}$ have a certain homogeneity property; see the discussion in [2, pp53-54]. Using the symmetry property $\beta_{5}(\Phi, \tilde{\Phi}, D)=\beta_{5}(\tilde{\Phi}, \Phi, \tilde{D})$, we see there exist constants so

$$
\begin{aligned}
\beta_{5}(\Phi, \tilde{\Phi}, D)= & \frac{1}{15 \sqrt{\pi}} \int_{\partial M}\left\{a_{1} \mathcal{P}_{; m m} \Phi \tilde{\Phi}+a_{2} \mathcal{P}_{; m}\left(\Phi_{; m} \tilde{\Phi}+\Phi \tilde{\Phi}_{; m}\right)\right. \\
& +a_{3} \mathcal{P}(D \Phi \cdot \tilde{\Phi}+\Phi \tilde{D} \tilde{\Phi})+a_{4} \mathcal{P} \Phi_{; m} \tilde{\Phi}_{; m}+a_{5} \mathcal{P}^{2} \Phi \tilde{\Phi} \\
& +a_{6}\left(D^{2} \Phi \cdot \tilde{\Phi}+\Phi \tilde{D}^{2} \Phi\right)+a_{7}\left\{(D \Phi)_{; m} \tilde{\Phi}_{; m}\right. \\
& \left.+\Phi_{; m}(\tilde{D} \Phi)_{; m}\right\}+a_{8} D \Phi \cdot \tilde{D} \Phi+a_{9} \mathcal{P}(\Phi ; a a \\
& \left.\tilde{\Phi}_{; 0}+\tilde{\Phi}_{; a a}\right) \\
& +a_{10} \mathcal{P} \Phi_{; a} \tilde{\Phi}_{; a}+a_{11} \Phi_{; a a} \tilde{\Phi}_{; b b}+a_{12}\left(\Phi_{; m m} \tilde{\Phi}_{; a a}\right. \\
& \left.\left.+\Phi_{; a a} \tilde{\Phi}_{; m m}\right)+a_{13} \Phi_{; m a} \tilde{\Phi}_{; m a}\right\}
\end{aligned}
$$

Product formulas then show the constants $a_{i}$ are independent of the dimension $m$.

We first take $k=0$ so $M=\left[r_{0}, r_{1}\right]$. Since $D \Phi=-\Phi_{; m m}-\mathcal{P} \Phi$, by Lemma 2.1

$$
\beta_{3}(\Phi, \tilde{\Phi}, D)=\frac{1}{3 \sqrt{\pi}} \int_{\partial M}\{4 D \Phi \cdot \tilde{\Phi}+4 \Phi \tilde{D} \tilde{\Phi}+2 \mathcal{P} \Phi \tilde{\Phi}\} .
$$

Suppose $\left.\Phi\right|_{\partial M}=0$. By Lemma $1.1, \beta_{5}(\Phi, \tilde{\Phi}, D)=-\frac{2}{5} \beta_{3}(D \Phi, \tilde{\Phi}, D)$. Thus we have

$$
\beta_{5}(\Phi, \tilde{\Phi}, D)=\frac{1}{15 \sqrt{\pi}} \int_{\partial M}\left\{-8 D^{2} \Phi \cdot \tilde{\Phi}-8 D \Phi \cdot \tilde{D} \tilde{\Phi}-4 \mathcal{P} D \Phi \cdot \tilde{\Phi}\right\}
$$

and hence $a_{2}=0, a_{3}=-4, a_{4}=0, a_{6}=-8, a_{7}=0$, and $a_{8}=-8$. Let $\Phi$ be general. We see that $a_{5}=1$ by using Lemma 1.1 and equation (3.2):

$$
\begin{aligned}
\beta_{3}(\Phi, \tilde{\Phi}, D) & =\left.\partial_{\epsilon}\right|_{\epsilon=0} \beta_{5}(\Phi, \tilde{\Phi}, D-\epsilon I) \\
& =\frac{1}{15 \sqrt{\pi}} \int_{\partial M}\left\{20 \Phi \cdot \tilde{\Phi}+20 \Phi \tilde{D} \tilde{\Phi}+8 \mathcal{P} \Phi \tilde{\Phi}+2 a_{5} \mathcal{P}\right\} .
\end{aligned}
$$

We have $d s_{e}^{2}=d r^{2}+r^{2} d s_{\theta}^{2}$ and $\Delta_{e}:=-\partial_{r}^{2}-(m-1) r^{-1} \partial_{r}+r^{-2} \Delta_{\theta}$ are the flat metric and Euclidean Laplacian in polar coordinates.

Let $M_{1}:=\left(r_{0}, r_{1}\right) \subset \mathbb{R}$ and let $M:=\left\{x: r_{0}<|x|<r_{1}\right\} \subset \mathbb{R}^{m}$. On $C^{\infty}\left(M_{1}\right)$, let $D:=-\partial_{r}^{2}-(m-1) r^{-1} \partial_{r}$. By Lemma 1.1 and Lemma 3.1,

$$
\begin{aligned}
& \beta_{5}\left(1,1, \Delta_{e}\right)=\beta_{5}\left(1, r^{m-1}, D\right) \operatorname{vol}\left(S^{m-1}\right) \\
& \quad=\frac{1}{240 \sqrt{\pi}}(m-1)(m-3)(m+3)(m-7)\left(r_{0}^{m-5}+r_{1}^{m-5}\right) \operatorname{vol}\left(S^{m-1}\right) .
\end{aligned}
$$


By Lemma $1.2, \omega=\frac{1}{2}(m-1) r^{-1}$ and $\mathcal{P}=-\frac{1}{4}(m-1)(m-3) r^{-2}$. Note $D(1)=0$ and $\tilde{D}\left(r^{m-1}\right)=0$. We show $a_{1}=1$ by equating coefficients in the equation

$$
\begin{aligned}
\frac{1}{15 \sqrt{\pi}}\left\{-\frac{3}{2} a_{1}(m-1)(m-3)+\right. & \left.\frac{1}{16}(m-1)^{2}(m-3)^{2}\right\} \\
& =\frac{1}{240 \sqrt{\pi}}(m-1)(m-3)(m+3)(m-7) .
\end{aligned}
$$

To evaluate the remaining coefficients, we take $k=1$ so $M=\left[r_{0}, r_{1}\right] \times S^{1}$. Let $D=D_{r}+D_{u}$ for $D_{r}=-\partial_{r}^{2}$ and $D_{u}=-\left(\partial_{u}^{2}+\mathcal{P}(u)\right)$.

Let $\Phi(r, u)=\phi(r) \psi(u)$ and $\tilde{\Phi}(r, u)=\tilde{\phi}(r) \tilde{\psi}(u)$. Because $u_{\Phi}=u_{\phi} u_{\psi}$, we have

$$
\beta_{n}(\Phi, \tilde{\Phi}, D)=\sum_{p+q=n} \beta_{p}\left(\phi, \tilde{\phi}, D_{r}\right) \beta_{q}\left(\psi, \tilde{\psi}, D_{u}\right)
$$

Since the boundary of the circle is empty,

$$
\beta_{2 k}\left(\psi, \tilde{\psi}, D_{u}\right)=(-1)^{k} \int_{S^{1}}\left(D_{u}^{k} \psi \cdot \tilde{\psi}\right) / k !
$$

and $\beta_{2 k+1}\left(\psi, \tilde{\psi}, D_{t}\right)=0$. We use equations (3.1), (3.3), and (3.4). By equating coefficients of the invariants $\mathcal{P} \Phi \cdot \tilde{\Phi}_{: a a}+\mathcal{P} \Phi_{; a a} \tilde{\Phi}, \mathcal{P} \Phi_{; a} \tilde{\Phi}_{; a}, \Phi_{; a a} \tilde{\Phi}_{; b b}$, $\Phi_{; m a} \tilde{\Phi}_{; m a}$, and $\Phi_{; m m} \tilde{\Phi}_{; a a}+\Phi_{; a a} \tilde{\Phi}_{; m m}$, we show that

$$
\begin{aligned}
& \frac{1}{15 \sqrt{\pi}}\left(a_{9}-20\right)=-\frac{2}{\sqrt{\pi}} \frac{1}{2}, \frac{1}{15 \sqrt{\pi}} a_{10}=0, \frac{1}{15 \sqrt{\pi}}\left(a_{11}-24\right)=-\frac{2}{\sqrt{\pi}} \frac{1}{2}, \\
& \frac{1}{15 \sqrt{\pi}} a_{13}=0, \quad \text { and } \quad \frac{1}{15 \sqrt{\pi}}\left(a_{12}-24\right)=-\frac{2}{\sqrt{\pi}} \frac{2}{3} .
\end{aligned}
$$

We complete the proof of the assertion (1) by solving these equations to see $a_{9}=5, a_{10}=0, a_{11}=9, a_{12}=4$, and that $a_{13}=0$. Assertion (2a) follows directly from Lemma 1.2. Assertion (2b) follows from assertion (1) since $1_{; a a}=\tilde{g}_{; a a}=-\mathcal{P}_{u}$ and $1_{; m m}=\tilde{g}_{; m m}=-\mathcal{P}_{r}$.

\section{The computation of $\mathcal{B}_{5}$.}

In this section, we complete the proof of Theorem 0.2 (5); by equation (1.3) it suffices to compute $\beta_{5}(1,1, \Delta)$. 
Lemma 4.1. (1) There exist universal constants so that

$$
\begin{aligned}
\beta_{5}\left(1,1, \Delta_{M}\right)= & \frac{1}{240 \sqrt{\pi}} \int_{\partial M}\left\{b_{1} \rho_{m m ; m m}+b_{2} L_{a a} \rho_{m m ; m}\right. \\
& +b_{3} L_{a b} R_{a m m b ; m}+b_{4} \rho_{m m}^{2}+b_{5} R_{a m m b} R_{a m m b} \\
& +b_{6} L_{a a} L_{b b} \rho_{m m}+b_{7} L_{a b} L_{a b} \rho_{m m}+b_{8} L_{a b} L_{a c} R_{m b c m} \\
& +b_{9} L_{a a} L_{b c} R_{m b c m}+b_{10} R_{a m m b} R_{a c c b}+b_{11} L_{a a} L_{b c} R_{b d d c} \\
& +b_{12} L_{a b} L_{a c} R_{b d d c}+b_{13} L_{a b} L_{c d} R_{a c b d}+b_{14} R_{a b c m} R_{a b c m} \\
& +b_{15} R_{a b b m} R_{a c c m}+b_{16} L_{a a: b} R_{b c c m}+e_{1} L_{a b: c} L_{a b: c} \\
& +e_{2} L_{a a} L_{b b} L_{c c} L_{d d}+e_{3} L_{a a} L_{b b} L_{c d} L_{c d}+e_{4} L_{a b} L_{a b} L_{c d} L_{c d} \\
& \left.+e_{5} L_{a a} L_{b c} L_{c d} L_{d b}+e_{6} L_{a b} L_{b c} L_{c d} L_{d a}\right\}
\end{aligned}
$$

(2) $e_{2}=1, e_{3}=-4, e_{4}+e_{5}=-20, e_{6}=48$.

(3) $b_{1}=8, b_{2}=-8, b_{3}=16, b_{4}=-4, b_{5}=16, b_{6}=-4, b_{7}=-8$, $b_{8}=64, b_{9}=-16, b_{10}=0, b_{11}=0, b_{12}=b_{13}, e_{4}=-4-b_{12}$, $e_{5}=-16+b_{12}$.

(4) $b_{12}=-8, b_{13}=-8, b_{14}=4, b_{15}=8, b_{16}=-16, e_{1}=-8$.

Proof. We use the Weyl calculus to write down a basis of invariants. We omit the invariants $\left\{\rho_{i i: a a}, \rho_{m m: a a}\right\}$ since they integrate to zero. We omit $\rho_{i i ; m m}$ and $\rho_{i i ; m} L_{a a}$ since we may express $\int_{\partial M} \rho_{i i ; m m}$ and $\int_{\partial M} \rho_{i i ; m} L_{a a}$ in terms of $\int_{\partial M} \rho_{m m ; m m}$ and $\int_{\partial M} \rho_{m m ; m} L_{a a}$ plus lower order terms. Since $R_{a b c m}=L_{b c: a}-L_{a c: b}$, we may symmetrize the covariant derivatives of $L$ modulo this tensor. We integrate by parts to avoid expressions in the second tangential covariant derivatives $L_{a b: c d}$ of $L$. We use product formulas to see the coefficients of the invariants $\rho_{i i}^{2},|\rho|^{2},|R|^{2}, \rho_{m m} \rho_{i i}, L_{a a} L_{b b} \rho_{i i}$, and $L_{a b} L_{a b} \rho_{i i}$ vanish. This proves the first assertion. If $M$ is the unit disk in $\mathbb{R}^{m}$, then by Lemma 1.1

$$
\begin{aligned}
e_{2}(m-1)^{4}+e_{3}(m-1)^{3}+\left(e_{4}+e_{5}\right) & (m-1)^{2}+e_{6}(m-1) \\
& =\left(m^{3}-7 m^{2}-9 m+63\right)(m-1) .
\end{aligned}
$$

The second assertion now follows.

We use the results of $\S 3$ to prove the remaining assertions. We take $k=0$ and consider the metric $d s^{2}=d r^{2}+e^{2 f_{i}(r, v)} d v_{i}^{2}$ on $\left[r_{0}, r_{1}\right] \times T^{\ell}$ as in Lemma 
3.1. Let ' $/$ ' denote partial differentiation. Let $m$ denote $\partial_{r}$; the remaining indices range from 1 through $\ell$ and index the $\partial_{a}^{v}$ derivatives. Then

$$
\begin{aligned}
R_{m a b m ; m} & =-\delta_{a b}\left(f_{a / m m}+f_{a / m}^{2}\right) / m \\
R_{m a b m} & =-\delta_{a b}\left(f_{a / m m}+f_{a / m m}^{2}\right) \\
R_{m a b m ; m m} & =-\delta_{a b}\left(f_{a / m m}+f_{a / m}^{2}\right) / m m \\
L_{a b} & =-\delta_{a b} f_{a / m}, \quad \text { and } \\
R_{a b c d} & =L_{a c} L_{b d}-L_{a d} L_{b c}+R_{a b c d}^{\partial M}
\end{aligned}
$$

We use equation (4.1) and sum over indices $a, b$, etc:

$$
\begin{aligned}
& b_{1} \rho_{m m ; m m}= b_{1}\left(-f_{a / m m m m}-2 f_{a / m m m} f_{a / m}-2 f_{a / m m}^{2}\right) \\
& b_{2} L_{a a} \rho_{m m ; m}= b_{2} f_{a / m}\left(f_{b / m m m}+2 f_{b / m m} f_{b / m}\right), \\
& b_{3} L_{a b} R_{a m m b ; m}= b_{3}\left(f_{a / m m m} f_{a / m}+2 f_{a / m m} f_{a / m}^{2}\right), \\
& b_{4} \rho_{m m}^{2}= b_{4}\left(f_{a / m m}+f_{a / m}^{2}\right)\left(f_{b / m m}+f_{b / m}^{2}\right), \\
& b_{5} R_{a m m b} R_{a m m b}= b_{5}\left(f_{a / m m}+f_{a / m}^{2}\right)^{2} \\
& b_{6} L_{a a} L_{b b} \rho_{m m}=-b_{6} f_{a / m} f_{b / m}\left(f_{c / m m}+f_{c / m}^{2}\right), \\
& b_{7} L_{a b} L_{a b} \rho_{m m}=-b_{7} f_{a / m}^{2}\left(f_{b / m m}+f_{b / m}^{2}\right), \\
& b_{8} L_{a b} L_{a c} R_{m b c m}=-b_{8} f_{a / m}^{2}\left(f_{a / m m}+f_{a / m}^{2}\right) \\
& b_{9} L_{a a} L_{b c} R_{m b c m}=-b_{9} f_{a / m} f_{b / m}\left(f_{b / m m}+f_{b / m}^{2}\right) \\
& b_{10} R_{a m m b} R_{a c c b}= b_{10}\left(f_{a / m m}+f_{a / m} f_{a / m}\right)\left(f_{a / m} f_{a / m}+\left(-f_{a / m} f_{c / m}\right.\right. \\
&\left.\left.+R_{a c c a}^{\partial M}\right)\right) \\
& b_{11} L_{a a} L_{b c} R_{b d d c}= b_{11}\left(f_{a / m} f_{b / m}^{3}-f_{a / m} f_{b / m}^{2} f_{d / m}\right. \\
&\left.+f_{a / m} f_{b / m} R_{b d d b}^{\partial M}\right) \\
& b_{12} L_{a b} L_{a c} R_{b d d c}= b_{12}\left(f_{a / m}^{4}-f_{a / m}^{3} f_{d / m}+f_{a / m}^{2} R_{a d d a}^{\partial M}\right) \\
& b_{13} L_{a b} L_{c d} R_{a c b d}= b_{13}\left(f_{a / m}^{2} f_{b / m}^{2}-f_{a / m}^{4}+f_{a / m} f_{b / m} R_{a b a b}^{\partial M}\right) \\
& e_{2} L_{a a} L_{b b} L_{c c} L_{d d}= e_{2} f_{a / m} f_{b / m} f_{c / m} f_{d / m} \\
& e_{3} L_{a a} L_{b b} L_{c d} L_{c d}= e_{3} f_{a / m} f_{b / m} f_{c / m}^{2} \\
& e_{4} L_{a b} L_{a b} L_{c d} L_{c d}= e_{4} f_{a / m}^{2} f_{b / m}^{2} \\
& e_{5} L_{a a} L_{b c} L_{c d} L_{d c}= e_{5} f_{a / m} f_{b / m}^{3}, \\
& e_{6} L_{a b} L_{b c} L_{c d} L_{d a}= e_{6} f_{a / m}^{4} . \\
& \text { and }
\end{aligned}
$$


The terms $b_{14} R_{a b c m} R_{a b c m}, b_{15} R_{a b b m} R_{a c c m}, b_{16} L_{a a: b} R_{b c c m}$, and $e_{1} L_{a b: c} L_{a b: c}$ will not play a role in this analysis. Let $\omega_{r}=\frac{1}{2} f_{a / m}, D=-\left(\partial_{r}^{2}+\omega_{r} \partial_{r}\right)$, and let $\mathcal{P}=-\frac{1}{4}\left(2 f_{a / m m}+\sum_{a, b} f_{a / m} f_{b / m}\right)$. We compute

$$
\begin{aligned}
16 \mathcal{P}_{; m m}+16 \mathcal{P}^{2}= & -8 f_{a / m m m m}+\left(-8 f_{a / m m m} f_{b / m}-4 f_{a / m m} f_{b / m m}\right) \\
& +4 f_{a / m m} f_{b / m} f_{c / m}+f_{a / m} f_{b / m} f_{c / m} f_{d / m} .
\end{aligned}
$$

Let $\omega_{r}=w_{r}(r)$. We use the results of $\S 3$ to equate the coefficients of the expressions

$$
\begin{array}{llll}
f_{a / m m} f_{a / m} f_{b / m}, & f_{a / m m m m}, & f_{a / m m} f_{a / m}^{2}, & \mathcal{H} f_{a / m m m}, \\
\mathcal{H} f_{a / m}^{3}, & \mathcal{H}^{2} f_{a / m m}, & f_{a / m m} f_{b / m}^{2}, & f_{a / m m} f_{b / m m}, \\
\mathcal{H}^{2} f_{a / m}^{2}, & f_{a / m m}^{2}, & \left(f_{a / m} f_{b / m}\right)^{2}, & f_{a / m m} R_{a c c a}^{\partial M}, \\
f_{a / m}^{4}, & \mathcal{H}^{4}, & f_{a / m m m} f_{a / m} &
\end{array}
$$

in the sum of terms from displays (4.2) and (4.3) to derive the equations

$$
\begin{aligned}
2 b_{2}-b_{9}-b_{10} & =0 \\
-b_{1} & =-8 \\
2 b_{3}+2 b_{5}-b_{8}+b_{10} & =0, \\
b_{2} & =-8, \\
-b_{9}-b_{10}-b_{11}-b_{12}+e_{5} & =0, \\
-b_{6} & =4, \\
2 b_{4}-b_{7} & =0, \\
b_{4} & =-4, \\
-b_{6}-b_{11}+e_{3} & =0, \\
-2 b_{1}+b_{5} & =0, \\
b_{4}-b_{7}+b_{13}+e_{4} & =0, \\
b_{10} & =0, \\
b_{5}-b_{8}+b_{10}+b_{12}-b_{13}+e_{6} & =0, \\
e_{2} & =1, \\
-2 b_{1}+b_{3} & =0 .
\end{aligned} \text { and }
$$

Assertion (3) now follows. As a scholium to our computations we see

$$
\mathcal{P}_{r}=\frac{1}{4}\left\{-2 f_{a / m m}-f_{a / m} f_{b / m}\right\}, \quad \text { and }
$$




$$
\begin{aligned}
& \beta_{5}\left(1,1, \Delta_{M}\right)-\frac{1}{240 \sqrt{\pi}} \int_{S^{1}}\left\{\left(16 \mathcal{P}_{r ; m m}+16 \mathcal{P}_{r}^{2}\right) \tilde{g}\right\} \\
& =\frac{1}{240 \sqrt{\pi}} \int_{\partial M}\left\{b_{12} L_{a b} L_{a c} R_{b d d c}^{\partial M}+b_{12} L_{a b} L_{c d} R_{a c b d}^{\partial M}+b_{14} R_{a b c m} R_{a b c m}\right. \\
& \left.\quad+b_{15} R_{a b b m} R_{a c c m}+b_{16} L_{a a: b} R_{b c c m}+e_{1} L_{a b: c} L_{a b: c}\right\}
\end{aligned}
$$

Next we take a metric of the form $d s_{M}^{2}=d r^{2}+d x^{2}+e^{2 f_{v}(r, x)} d v_{j}^{2}$. Let $m$ denote $\partial_{r}$ and 1 denote $\partial_{x}$. The remaining indices $u, v$ will range from 2 through $m-1$ and index the $\partial_{v}^{j}$ derivatives. We sum over $u$, $v$, etc. and compute

$$
\begin{aligned}
& \mathcal{P}_{u}=-\frac{1}{2} f_{u / m m}-\frac{1}{4} f_{u / m} f_{v / m} \\
& R_{u 1 m v}=\delta_{u v}\left(-f_{u / 1 m}-f_{u / 1} f_{u / m}\right) \\
& R_{u 11 v}=\delta_{u v}\left(-f_{u / 11}-f_{u / 1}^{2}\right) \\
& R_{u v v u}^{\partial M}=\delta_{u v} f_{u / 1}^{2}-f_{u / 1} f_{v / 1} \\
& b_{12} L_{a b} L_{a c} R_{b d d c}^{\partial M}=b_{12} f_{u / m}^{2}\left(-f_{u / 11}-f_{u / 1}^{2}\right)+f_{u / m}^{2}\left(\delta_{u v} f_{u / 1}^{2}-f_{u / 1} f_{v / 1}\right) \\
& b_{13} L_{a b} L_{c d} R_{a c b d}^{\partial M}=b_{13} f_{u / m} f_{v / m}\left(f_{u / 1} f_{v / 1}-\delta_{u v} f_{u / 1}^{2}\right) \\
& b_{14} R_{a b c m} R_{a b c m}=2 b_{14} R_{1 u u m}^{2}=2 b_{14}\left(f_{u / 1 m}+f_{u / 1} f_{u / m}\right)^{2} \\
& b_{15} R_{a b b m} R_{a c c m}=b_{15}\left(f_{u / 1 m}+f_{u / 1} f_{u / m}\right)\left(f_{v / 1 m}+f_{v / 1} f_{v / m}\right) \\
& b_{16} L_{a a: b} R_{b c c m}=b_{16} f_{u / 1 m}\left(f_{v / 1 m}+f_{v / 1} f_{v / m}\right) \\
& e_{1} L_{a b: c} L_{a b: c}=\left(f_{u / 1 m}^{2} f_{u / 1 m}+f_{u / m}^{2} f_{u / 1}^{2}\right) \\
& 16 \mathcal{P}_{u ; m m}=-8 f_{u / 11 m m}-8\left(f_{u / 1 m} f_{v / 1 m}+f_{u / 1 m m} f_{v / 1}\right) \\
& \int_{r_{0} \times S_{1}}\left\{16 g \mathcal{P}_{u ; m m}\right\}=\int_{\partial M}\left\{b_{12} L_{a b} L_{a c} R_{b d d c}^{\partial M}+b_{12} L_{a b} L_{c d} R_{a c b d}^{\partial M}\right. \\
&\left.+b_{14} R_{a b c m} R_{a b c m}+b_{15} R_{a b b m} R_{a c c m}+b_{16} L_{a a: b} R_{b c c m}+e_{1} L_{a b: c} L_{a b: c}\right\} \\
& \int_{\partial M}\left\{-b_{12} f_{u / m}^{2} f_{u / 11}-b_{12} f_{u / m}^{2} f_{u / 1} f_{v / 1}\right\}=\int_{\partial M} 2 b_{12} f_{u / 1 m} f_{u / 1} f_{u / m},
\end{aligned}
$$

and

$$
\int_{r_{0} \times S^{1}}\left\{e^{f_{u}}\left(-8 f_{u / 11 m m}-8 f_{u / 11 m} f_{v}\right)\right\}=0 .
$$

There are no other integral identities among the monomials involved. We equate coefficients of $f_{u / 1 m} f_{v / 1} f_{v / m}, f_{u / 1 m} f_{v / 1 m}, f_{u / 1 m} f_{u / 1} f_{u / m}, f_{u / 1 m}^{2}$, 
$f_{u / m} f_{v / m} f_{u / 1} f_{v / 1}$, and $f_{u / m}^{2} f_{u / 1}^{2}$ to derive the following equations:

$$
\begin{aligned}
& 2 b_{15}+b_{16}=0, \quad b_{15}+b_{16}=-8, \quad 2 b_{12}+4 b_{14}=0, \\
& 2 b_{14}+e_{1}=0, \quad b_{13}+b_{15}=0, \text { and }-b_{13}+2 b_{14}+2 e_{1}=0 \text {. }
\end{aligned}
$$

We use these equations and the preceding assertions to complete the proof.

\section{References.}

[1] M. van den Berg, S. Desjardins, and P. Gilkey, Functoriality and heat content aymptotics for operators of Laplace type, Topological Methods in Nonlinear Analysis, 2 (1993), 147-162.

[2] M. van den Berg and P. Gilkey, Heat Content Asymptotics of a Riemannian Manifold with Boundary, Journal of Functional Analysis, 120 (1994), 48-71.

[3] M. van den Berg and J-F Le Gall, Mean curvature and the heat equation, Math. Z. 215 (1994), 437-464.

[4] H.S. Carslaw and J.C. Jaeger, Conduction of Heat in Solids, Oxford Science Publications, Oxford University Press, 1946.

[5] S. Desjardins and P. Gilkey, Heat content asymptotics for operators of Laplace type with Neumann boundary conditions, Math. Z. 215 (1994), 251-268.

[6] P. Gilkey, The spectral geometry of a Riemannian manifold, J. Diff. Geo. 10 (1975), 601-618.

[7] D.M. McAvity, Heat kernel asymptotics for mixed boundary conditions, Class. Quant. Grav. 9 (1992), 1983-1998.

[8] D.M. McAvity, Surface energy from heat content asymptotics, J. Phys. A.: Math. Gen. 26 (1993), 823-830.

RECEIVED JANUARY 17, 1997.

UNIVERSITY OF BRISTOL, UNIVERSITY WALK, BRISTOL BS8 1TW, U.K.

E-mail address: M.vandenBerg@bris.ac.uk 
AND

UNIVERSITY OF OREGON, EUGENE OR 97403 USA

E-mail address: gilkey@math.uoregon.edu 